\title{
A importância da avaliação nutricional no controle da dieta de uma equipe de jogadores de futebol juniores
}

\author{
Luciana Collet Winther Rebello ${ }^{1}$, Paulo Roberto Santos Silva ${ }^{2}$, Alberto Azevedo Alves Teixeira ${ }^{3}$, \\ José Roberto Rivelino Vidal ${ }^{4}$, Alexandre Augusto Ribeiro Barros Oberg ${ }^{4}$, Alcides Fonseca Jr. ${ }^{5}$, \\ Francisco Oliveira Rocha ${ }^{6}$ e Jorge Mendes Sousa ${ }^{7}$
}

Centro de Medicina Integrada da Associação Portuguesa de Desportos, SP, Brasil

\section{RESUMO}

O objetivo deste estudo foi verificar por meio de questionário alimentar (recordatório de 24 horas) a adequação da ingestão calórica diária de macronutrientes em 19 jogadores de futebol juniores, com média de idade de 18,6 \pm 1,1 anos (1620).

Todos os atletas eram pertencentes ao Departamento de Futebol Amador da Associação Portuguesa de Desportos de São Paulo. Os futebolistas foram submetidos a inquérito alimentar por meio de entrevista individualizada. $\mathrm{O}$ peso ideal dos atletas foi calculado por meio da média do índice de massa corpórea $(\mathrm{IMC})=22,5 \mathrm{~kg} \cdot \mathrm{m}^{2}$ multiplicado pela estatura em metros ao quadrado. A partir do resultado foi calculada a taxa metabólica basal utilizando-se a tabela da FAO/OMS/ONU. A seguir, por anamnese, realizada junto ao setor de preparação atlética, quantificou-se o volume de atividade física, que possibilitou determinar o valor calórico mínimo, médio e máximo adequado aos atletas. Além disso, todos os futebolistas foram submetidos a exames laboratoriais.

Os seguintes resultados e parâmetros foram verificados: o cálculo das necessidades calóricas diária de acordo com o volume de treinamento foi: mínimo $=3.298 \pm 177 \mathrm{kcal} . \mathrm{dia}^{-1}$;

1. Nutricionista.

2. Fisiologista.

3. Médico do Esporte.

4. Fisicultor.

5. Técnico de Futebol.

6. Anestesiologista.

7. Vice-Presidente de Medicina da Associação Portuguesa de DesportosSP.

Endereço para correspondência:

Centro de Medicina Integrada

Luciana Collet Winther Rebello (Nutricionista)

Rua Comendador Nestor Pereira, 33

03034-070 - São Paulo, SP - Brasil

Tel. (011) 225-0400, ramais 224/228

Fax (011) 228-8449 médio $=3.689 \pm 207 \mathrm{kcal}$. dia $^{-1}$ e máximo $=3.991 \pm 225 \mathrm{kcal}$. $\operatorname{dia}^{-1}$. O consumo calórico médio dos jogadores era de 2.584 $\pm 421 \mathrm{kcal} \mathrm{dia}^{-1}$.

Quando se comparou o resultado da ingestão média consumida pelos atletas com os valores mínimo, médio e máximo necessários, a deficiência calórica percentual apresentada, de $22 \%, 30 \%$ e $35 \%$, respectivamente, foi altamente significante ( $\mathrm{p}<0,05)$. Os exames laboratoriais mostraram, em um atleta, anemia estabelecida, com $\mathrm{Hb}$ de 11,1g\% e hematócrito de $35 \%$.

$\mathrm{O}$ resultado deste estudo permite concluir que não somente a educação nutricional visando corrigir desordens de comportamento alimentares, como também um controle rigoroso das necessidades calóricas, constituem procedimentos de importância fundamental para uma dieta adequada em jogadores de futebol.

Palavras-chave: Nutrição esportiva. Atividade física. Jogadores de futebol. Medicina Esportiva.

\section{ABSTRACT \\ Importance of nutritional evaluation for diet control in a team of junior soccer players}

The aim of this study was to verify, through a food questionnaire covering a 24-hour recollection range, the adequacy of the daily caloric intake of macronutrients in 19 soccer players whose mean age was $18.6 \pm 1.1$ (16-20).

All athletes belonged to the Amateur Soccer Department of Associação Portuguesa de Desportos in São Paulo. The soccer players were submitted to a food inquiry by means of personalized interviews. The ideal weight of the athletes was calculated by the mean body mass index $(B M I)=22.5 \mathrm{~kg} \cdot \mathrm{m}^{2}$ multiplied by the stature in square meters. The basal metabolic rate was then calculated, the FAO/WHO/UN chart having been used. After that, by anamnesis carried out at the sector of athletic preparation, the volume of physical activity was quantified, which made it possible to determine the minimum, mena and maximum caloric value suitable for athletes. In addition, all soccer players were submitted to laboratory tests. 
By calculating the daily caloric needs according to the training, the following result and parameters were verified: minimum $=3,298 \pm 177 \mathrm{kcal}$.day ${ }^{-1}$; mean $=3,689 \pm 207 \mathrm{kcal}$. day $^{-1}$ and maximum $=3,991 \pm 225 \mathrm{kcal}^{\text {.day }}{ }^{-1}$. The mean caloric consumption reached by the players used to be 2,584 \pm $421 \mathrm{kcal}^{-d a y^{-1}}$. When a comparison was made between the mean intake they consumed with the minimal, mean and maximal values necessary, the percentage of caloric deficiency which they presented $-22 \%, 30 \%$ and $35 \%$, respectively was highly significant $(p<0.05)$. The laboratory tests revealed in one of the athletes the presence of anemia with $11.1 \%$ of $\mathrm{Hb}$ and $35 \%$ of hematocrit.

The results of this study allow to conclude that both nutritional education intended to correct food behavior disorders, and severe control of the caloric needs, constitute procedures of prime importance for an adequate diet of soccer players.

Key words: Sports nutrition. Physical activity. Soccer players. Sports Medicine.

\section{INTRODUÇÃO}

A nutrição voltada para o esporte de alto rendimento é, na atualidade, uma das áreas que mais têm recebido atenção e valorização pelos profissionais do esporte ${ }^{1-5}$. Isso justifica-se, porque, quando a dieta é prescrita de maneira correta, pode otimizar os depósitos de energia para a competição, o que pode ser a diferença no resultado final em atividades que demandem volume e variadas intensidades ${ }^{5}$.

Além disso, traz benefícios importantes para a saúde orgânica do atleta, diminuindo as possibilidades de doenças que possam afetar seu condicionamento físico. A dieta pode influenciar seu desempenho atlético e a confiança no consumo de certos alimentos pode conferir uma vantagem psicológica particularmente importante para o atleta.

Atletas submetidos a constante treinamento e a grandes volumes de atividade física intensa têm requerimentos nutricionais diferentes quando comparados com indivíduos não atletas.

Contudo, não basta o atleta comer qualquer alimento; é necessário controlar o volume, a qualidade do nutriente e seu ajuste às necessidades calóricas da atividade física.
A preparação e a orientação adequadas da ingestão de macro e micronutrientes são essenciais para a realização de várias ações biológicas do organismo submetido a treinamento desportivo ${ }^{6}$.

A influência do alimento é determinante para o funcionamento metabólico adequado ao organismo do atleta às solicitações de demanda física impostas pelo exercício físico durante a realização de treinamentos e competições.

Portanto, com todos esses benefícios, fica fácil compreender por que a nutrição tem recebido tamanha atenção dos profissionais que militam no esporte.

Entretanto, é importante salientar que a nutrição é parte de um todo, ela não faz milagre, não interfere no fator genético para a prática de esporte e não substitui o treinamento do atleta; ela apenas contribui com a melhora do organismo para realizar exercícios, de acordo com as necessidades energéticas da atividade física.

O principal objetivo deste estudo foi verificar, por meio de questionário alimentar, adequação da ingestão calórica diária dos macronutrientes em futebolistas juniores.

\section{MATERIAL E MÉTODOS}

Foram submetidos à avaliação nutricional por meio de inquérito alimentar (recordatório de 24 horas) 19 jogadores de futebol, categoria juniores, pertencentes ao Departamento de Futebol Amador da Associação Portuguesa de Desportos de São Paulo. Todos eram do sexo masculino, com idade variando de 16 a 20 anos. Além disso, foram calculados os índices de massa e superfície corpórea (IMC e SC), respectivamente (tabela 1).

O peso ideal dos futebolistas foi calculado por meio da média do valor do $\mathrm{IMC}=22,5 \mathrm{~kg} \cdot \mathrm{m}^{2}$ multiplicado pela estatura ao quadrado em metros. A partir do resultado calculou-se a taxa metabólica basal utilizando-se a tabela da FAO/OMS/ONU, $1985^{7}$.

A seguir, pela anamnese realizada junto ao setor de preparação atlética, quantificou-se a atividade física, que possibilitou determinar o valor calórico mínimo, médio e máximo necessário a ser atingido pelos atletas (tabela 2). A partir daí, calculou-se a percentagem de ingestão e a possível deficiên-

TABELA 1

Características físicas e hematológicas dos jogadores de futebol $(n=19)$

\begin{tabular}{|c|c|c|c|c|c|c|}
\hline $\begin{array}{l}\text { Idade } \\
\text { (anos) }\end{array}$ & $\begin{array}{l}\text { Peso } \\
\text { (kg) }\end{array}$ & $\begin{array}{l}\text { Estatura } \\
\text { (cm) }\end{array}$ & $\begin{array}{c}\text { IMC } \\
\left(\mathbf{k g} \cdot \mathrm{m}^{2}\right)\end{array}$ & $\begin{array}{c}S C \\
\left(m^{2}\right)\end{array}$ & $\begin{array}{l}\text { Hb } \\
(\mathbf{g} \%)\end{array}$ & $\begin{array}{c}\text { Hematócrito } \\
\text { (\%) }\end{array}$ \\
\hline $\begin{array}{c}18,6 \\
\pm 1,1 \\
(16-20)\end{array}$ & $\begin{array}{c}70,4 \\
\pm 8,4 \\
(55,5-96,5)\end{array}$ & $\begin{array}{c}175,5 \\
\pm 7,4 \\
(164-194)\end{array}$ & $\begin{array}{c}22,90 \\
\pm 1,33 \\
(20,8-24,7)\end{array}$ & $\begin{array}{c}1,86 \\
\pm 0,11 \\
(1,60-2,02)\end{array}$ & $\begin{array}{c}14,2 \\
\pm 1,0 \\
(11,1-17,1)\end{array}$ & $\begin{array}{c}42,8 \\
\pm 3,1 \\
(35-46)\end{array}$ \\
\hline
\end{tabular}

Os resultados representam a média, o desvio-padrão e as variações mínima e máxima 
cia calórica geral e individual em relação à média necessária para o grupo de futebolistas (tabelas 3 e 4). Além disso, todos os futebolistas foram submetidos a exames laboratoriais (tabela 5).

\section{Análise estatística}

Os dados foram analisados utilizando-se o cálculo da média, desvio-padrão e as variações mínima e máxima. Para comparar a diferença entre a ingestão calórica consumida e a ideal, foi utilizada análise de variância (ANOVA) de um caminho e, em caso de F significante entre as médias, foi aplicado o teste post hoc de Bonferroni com nível de significância de $\mathrm{p}<0,05^{8}$.

\section{TABELA 2}

Projeção das necessidades calóricas mínima, média e máxima de acordo com o volume de treinamento realizado pelos jogadores de futebol avaliados - categoria juniores ${ }^{8}(n=19)$

\begin{tabular}{rrrr}
\hline $\begin{array}{c}\text { No } \\
\text { do atleta }\end{array}$ & $\begin{array}{c}\text { Mínima } \\
\text { (kcal.dia-1) }\end{array}$ & $\begin{array}{c}\text { Média } \\
\text { (kcal.dia-1) }\end{array}$ & $\begin{array}{c}\text { Máxima } \\
\text { (kcal.dia-1) }\end{array}$ \\
1 & 3.331 & 3.919 & 4.252 \\
2 & 3.269 & 3.846 & 4.173 \\
3 & 3.097 & 3.442 & 3.734 \\
4 & 3.230 & 3.589 & 3.894 \\
5 & 3.423 & 3.804 & 4.127 \\
6 & 3.414 & 3.793 & 4.116 \\
7 & 3.130 & 3.478 & 3.774 \\
8 & 3.732 & 4.146 & 4.499 \\
9 & 3.462 & 3.846 & 4.173 \\
10 & 3.032 & 3.369 & 3.656 \\
11 & 3.353 & 3.726 & 4.043 \\
12 & 3.174 & 3.527 & 3.827 \\
13 & 3.220 & 3.687 & 3.882 \\
14 & 3.319 & 3.662 & 4.001 \\
15 & 3.295 & 3.781 & 3.973 \\
16 & 3.402 & 3.551 & 3.853 \\
17 & 3.196 & 3.939 & 4.274 \\
18 & 3.545 & 3.614 & 3.921 \\
19 & 3.043 & 3.381 & 3.668 \\
\hline
\end{tabular}

Os cálculos representam os valores em calorias individuais para cada futebolista

\section{RESULTADOS}

Os resultados deste estudo estão listados nas tabelas 1, 2, 3, 4 e 5 .

\section{COMENTÁRIOS E DISCUSSÃO}

A nutrição para o esporte tem dado suporte importante para a melhora do rendimento físico de atletas ${ }^{9-11}$. A verificação de hábitos alimentares é uma estratégia adequada, pois permite identificar possíveis deficiências e/ou desequilíbrios dos nutrientes na dieta para realização da atividade física ${ }^{12}$.

Entretanto, a falta de educação nutricional no meio esportivo tem sido a principal dificuldade encontrada para conscientizar atletas e técnicos sobre o tipo de alimentação mais apropriada à demanda energética imposta por sua atividade desportiva específica. Sabe-se que a compensação do gasto energético, durante e após competição, só irá acontecer na medida em que os depósitos de energia forem preenchidos adequadamente.

A Seção de Nutrição do Centro de Medicina Integrada da Associação Portuguesa de Desportos de São Paulo, recémimplantada, vem dando atenção especial a seus atletas, com a monitorização de seus hábitos alimentares em busca da conscientização sobre a ingestão de alimentos adequados às necessidades de um atleta futebolista.

Os resultados verificados em nosso estudo, realizado com jogadores juniores, demonstraram as dificuldades que os atletas têm em se alimentar com qualidade. Falta consciência de que eles não são pessoas comuns, eles são indivíduos que solicitam elevadas taxas metabólicas durante treinamentos e jogos competitivos, precisam render o tempo todo e, portanto, necessitam um suporte maior desta área.

Alguns estudos têm verificado em jogadores de futebol o consumo calórico diário para suportarem o treinamento e a manutenção do peso corpóreo.

Maughan, citado por $\mathrm{Clark}^{6}$, reportou em dois estudos, realizados com futebolistas escoceses, ingestão de calorias entre 2.033 e $3.846 \mathrm{kcal}_{\text {dia }}{ }^{-1}$ e 2.044 e $3.923 \mathrm{kcal} \mathrm{dia}^{-1}$, respectivamente.

\begin{tabular}{|c|c|c|c|}
\hline \multicolumn{4}{|c|}{$\begin{array}{c}\text { TABELA } 3 \\
\text { Resultados comparativos do cálculo médio necessário e consumido, } \\
\text { percentagem e deficiência de calorias verificadas em um grupo } \\
\text { de jogadores de futebol - categoria juniores }(n=19)\end{array}$} \\
\hline $\begin{array}{l}\text { Consumo médio } \\
\text { necessário de } \\
\text { calorias (kcal.dia-1) }\end{array}$ & $\begin{array}{l}\text { Consumo } \\
\text { médio } \\
(\%)\end{array}$ & $\begin{array}{l}\text { Consumo médio } \\
\text { ingerido de } \\
\text { calorias (kcal.dia-1) }\end{array}$ & $\begin{array}{l}\text { Consumo } \\
\text { médio } \\
\text { (\%) }\end{array}$ \\
\hline $\begin{array}{l}3.689 * \\
\pm 207 \\
(3.381-4.146)\end{array}$ & $\begin{array}{l}70^{*} \\
\pm 11 \\
(49-88)\end{array}$ & $\begin{array}{c}2.584 \\
\pm 421 \\
(1.707-3.216)\end{array}$ & $\begin{array}{r}30 \\
\pm 11 \\
(12-55)\end{array}$ \\
\hline
\end{tabular}


TABELA 4

Resultado individual do cálculo calórico médio necessário, consumido, percentagem e deficiência de ingestão alimentar detectada em uma equipe de jogadores de futebol - categoria juniores $(n=19)$

\begin{tabular}{|c|c|c|c|c|}
\hline $\begin{array}{c}\text { № } \\
\text { do } \\
\text { atleta }\end{array}$ & $\begin{array}{l}\text { Média de } \\
\text { calorias } \\
\text { necessárias } \\
(\text { kcal.dia-1) }\end{array}$ & $\begin{array}{l}\text { Média de } \\
\text { calorias } \\
\text { consumidas } \\
\text { (kcal.dia-1) }\end{array}$ & $\begin{array}{c}\text { \% de ingestão } \\
\text { calórica diária } \\
\text { verificada } \\
\text { nos atletas }\end{array}$ & $\begin{array}{c}\text { \% de deficiência } \\
\text { calórica verificada } \\
\text { nos atletas }\end{array}$ \\
\hline
\end{tabular}

\begin{tabular}{rrrrl}
1 & 3.919 & 2.009 & 52 & 47 \\
2 & 3.846 & 3.216 & 83 & 16 \\
3 & 3.442 & 1.707 & 49 & 50 \\
4 & 3.589 & 2.391 & 65 & 34 \\
5 & 3.804 & 2.451 & 64 & 35 \\
6 & 3.793 & 1.708 & 45 & 54 \\
7 & 3.478 & 2.720 & 78 & 21 \\
8 & 4.146 & 2.991 & 72 & 27 \\
9 & 3.846 & 2.391 & 67 & 37 \\
10 & 3.369 & 2.969 & 88 & 11 \\
11 & 3.726 & 2.914 & 78 & 21 \\
12 & 3.527 & 2.465 & 69 & 30 \\
13 & 3.687 & 2.511 & 68 & 31 \\
14 & 3.662 & 2.610 & 71 & 28 \\
15 & 3.781 & 2.730 & 72 & 27 \\
16 & 3.551 & 2.880 & 81 & 18 \\
17 & 3.939 & 2.937 & 74 & 25 \\
18 & 3.614 & 2.954 & 81 & 18 \\
19 & 3.381 & 2.543 & 75 & 24 \\
\hline Cálculo baseado no recordatório de 24 horas & &
\end{tabular}

TABELA 5

Resultados individuais dos exames laboratoriais realizados pelos jogadores de futebol - categoria juniores $(n=19)$

\begin{tabular}{|c|c|c|c|c|c|c|}
\hline $\begin{array}{l}\text { № do } \\
\text { atleta }\end{array}$ & $\begin{array}{l}\text { Idade } \\
\text { (anos) }\end{array}$ & $\begin{array}{c}\text { Hb } \\
(g \%)\end{array}$ & $\begin{array}{c}\text { Eritrócitos } \\
\text { (milhões } / \mathbf{m m}^{3} \text { ) }\end{array}$ & $\begin{array}{c}\text { Hematócrito } \\
\text { (\%) }\end{array}$ & $\begin{array}{l}\text { Leucócitos } \\
\left(\mathbf{m i l} / \mathbf{m m}^{3}\right)\end{array}$ & $\begin{array}{l}\text { Plaquetas } \\
\left(\mathbf{m i l} / \mathbf{m m}^{3}\right)\end{array}$ \\
\hline 1 & 19 & 14,2 & 4,9 & 44 & 5,4 & 258 \\
\hline 2 & 19 & 11,1 & 4,7 & 35 & 6,3 & 309 \\
\hline 3 & 19 & 15,1 & 5,4 & 46 & 6,4 & 244 \\
\hline 4 & 19 & 14,3 & 4,5 & 42 & 7,2 & 261 \\
\hline 5 & 19 & 13,8 & 5,7 & 42 & 6,1 & 203 \\
\hline 6 & 20 & 13,9 & 4,5 & 42 & 8,6 & 279 \\
\hline 7 & 19 & 14,2 & 4,8 & 43 & 6,0 & 218 \\
\hline 8 & 20 & 13,7 & 4,5 & 40 & 7,6 & 225 \\
\hline 9 & 20 & 14,6 & 4,8 & 41 & 5,8 & 200 \\
\hline 10 & 19 & 14,9 & 5,0 & 43 & 5,5 & 244 \\
\hline 11 & 18 & 14,2 & 4,7 & 42 & 6,8 & 195 \\
\hline 12 & 20 & 14,1 & 4,5 & 41 & 7,9 & 198 \\
\hline 13 & 19 & 14,7 & 5,2 & 45 & 3,8 & 258 \\
\hline 14 & 18 & 14,3 & 4,9 & 44 & 7,3 & 294 \\
\hline 15 & 17 & 13,7 & 5,2 & 42 & 5,4 & 163 \\
\hline 16 & 17 & 14,0 & 4,5 & 42 & 6,4 & 209 \\
\hline 17 & 17 & 15,3 & 5,1 & 46 & 7,4 & 196 \\
\hline 18 & 16 & 14,1 & 4,9 & 43 & 5,9 & 239 \\
\hline 19 & 19 & 17,1 & 5,5 & 51 & 7,0 & 283 \\
\hline
\end{tabular}


Caldarone et al. ${ }^{13}$, estudando 33 jogadores de futebol da primeira divisão italiana, verificaram valor médio de $3.066 \pm$ $568 \mathrm{kcal} \mathrm{dia}^{-1}$. Enquanto isso, Reilly ${ }^{14}$ reportou, num futebolista inglês de nível internacional, ingestão calórica de 3.127 kcal.dia ${ }^{-1}$.

No presente estudo, calculamos para os atletas a ingestão mínima de calorias necessárias (3.298 $\mathrm{kcal}$ dia $\left.^{-1}\right)$, a média (3.689kcal.dia ${ }^{-1}$ ) e a máxima (3.991 kcal.dia $\left.{ }^{-1}\right)$. Quando comparamos com a ingestão média que vinham fazendo $(2.584$ kcal.dia ${ }^{-1}$ ), a deficiência ficou clara: $22 \%, 30 \%$ e 35\%, respectivamente.

Também comparamos os resultados da ingestão alimentar média do grupo, realizada diariamente no clube, ou seja, 2.584 kcal.dia ${ }^{-1}$, com a média necessária calculada $(3.689 \mathrm{kcal}$. $\mathrm{dia}^{-1}$ ) verificada pelo questionário alimentar; a diferença foi altamente significante $(\mathrm{p}<0,05)$ [tabela 3]. Além disso, verificamos, em dois futebolistas, consumo extremamente baixo para um atleta, ou seja, somente $50 \%$ e $54 \%$ de ingestão calórica diária em relação ao necessário.

É importante salientar que a alimentação deficiente é uma das principais causas de diminuição do rendimento físico no atleta. Pois, uma das alterações orgânicas mais freqüentes é a anemia por deficiência de ferro. Além disso, a perda de ferro corpóreo é de aproximadamente $1,0 \mathrm{mg} \cdot \mathrm{dia}^{-1}$. Esse mineral é um componente importante da hemoglobina $(\mathrm{Hb})$, mioglobina e de enzimas da cadeia respiratória (citocromos e a citocromo-oxidase) e exerce papel fundamental na produção de energia $^{15}$. Ele é transportado no sangue ligado à proteína plasmática transferrina e nos tecidos é armazenado na forma de ferritina, constituindo-se em excelente marcador de seus depósitos.

É importante salientar que o ferro não é excretado na urina, mas é perdido pelo organismo na bile, fezes e no sangue menstrual. Portanto, pelo fato de a velocidade de perda do ferro no organismo feminino ser duplicada ou triplicada durante a menstruação, as mulheres requerem maiores quantidades dele que os homens.

A anemia do esporte é uma das teorias que tentam explicar a deficiência de ferro provocada pelo treinamento físico excessivo, sendo uma das causas que predispõem à deficiência de ferro. Dentre estas, podemos citar: 1) hemólise traumáti$\mathrm{ca}^{16}$; 2) pobre ingestão e redução de ferro ${ }^{17} ; 3$ ) hematúria ${ }^{18}$; e 4) adaptações fisiológicas do exercício ${ }^{19}$.

Têm sido considerados como variação normal da $\mathrm{Hb}$, em homens, valores entre 14 e $18 \mathrm{~g} . \mathrm{dl}^{-1}$, sendo $16 \mathrm{~g} \cdot \mathrm{dl}^{-1} \mathrm{o}$ valor médio aproximado para a população ${ }^{20}$. Segundo Pate ${ }^{20}$, concentração de $\mathrm{Hb}$ em atletas menor que $14 \mathrm{~g} \cdot \mathrm{dl}^{-1}$ é classificada como anemia pré-latente.

Os resultados deste estudo mostraram que, dos 19 atletas submetidos a exames laboratoriais, um caso apresentou anemia estabelecida com $\mathrm{Hb}$ de $11,1 \mathrm{~g} \%$ e o hematócrito de apenas $35 \%$. Outros quatro atletas estavam com valores abaixo de $14 \mathrm{~g} \%$ e não inferiores a $13 \mathrm{~g} \%$, este considerado em atletas como anemia pré-latente (tabela 5).

Considerando que a grande maioria de nossos atletas é proveniente de famílias humildes e, portanto, com poucos recursos, a realização de exames laboratoriais é de fundamental importância nesses futebolistas.

Esses resultados reforçam, ainda mais, a importância da função exercida pelo profissional especializado em nutrição esportiva, pois o controle e a ingestão da dieta adequada, o conhecimento dos hábitos e gostos alimentares são estratégias que objetivam fornecer ao atleta quantidade, qualidade e equilíbrio às necessidades calóricas de cada futebolista.

É importante lembrar que a garantia de uma nutrição adequada é verificada por uma dieta altamente variada junto com um balanço apropriado de calorias em relação às necessidades individuais, considerando a altura, o peso corpóreo e o grau de atividade física do indivíduo.

Contudo, não podemos esquecer que o folclore alimentar é cheio de histórias, atribuindo a determinados tipos de alimentos verdadeiros milagres. Em atletas, pouco tempo atrás, era aconselhável, no café da manhã, antes da competição esportiva, o consumo de apenas ovos e carnes, ricos em proteínas. Entretanto, as proteínas não são armazenadas e, portanto, não são úteis como fonte de energia como são os carboidratos.

Assim, do ponto de vista bioquímico, faria mais sentido preparar-se para uma atividade física empanturrando-se com carboidratos, como fazem os maratonistas, para elevar os níveis de glicogênio muscular e hepático, pois este é o combustível primordial para a produção de energia durante uma atividade física.

Portanto, a nutrição contemporânea voltada para o esporte tem a missão de acabar com os folclores alimentares, obsessões irracionais e poderes miraculosos atribuídos aos alimentos, orientando e garantindo aos atletas uma dieta calórica apropriada a suas necessidades metabólicas. Para atingir este objetivo, a educação nutricional corrigindo desordens de comportamento alimentar e o controle rigoroso das necessidades calóricas são mecanismos fundamentais para adequação da dieta em jogadores de futebol.

\section{AGRADECIMENTOS}

Gostaríamos de agradecer ao professor de língua inglesa Humberto Blancato pela tradução do Abstract deste manuscrito.

\section{REFERÊNCIAS}

1. Bergstrom J, Hermansen L, Hultman E, Saltin B. Diet muscle glycogen and physical performance. Acta Physiol Scand 1967;71:140-50.

2. Muckle DS. Glucose syrup ingestion and team performance in soccer. Br J Sports Med 1973;7:340-3.

3. Jacobs I, Westlin N, Rasmusson M, Hougthon B. Muscle glycogen and diet in elite soccer players. Eur J Appl Physiol 1982;48:297-302.

4. Evans WJ, Hughes VA. Dietary carbohydrates and endurance exercise. Am J Clin Nutr 1985;44:1146-54. 
5. Costill DL. Carbohydrate nutrition before, during and after exercise Federation Proc 1985;44:364-8.

6. Clark K. Nutritional guidance to soccer players for training and competition. J Sports Sci 1994;12:S43-S50.

7. Necessidades de energia e proteínas; informe de uma reunião consultiva conjunta FAO/OMS/ONU de experts - Genebra 1985 (Séries de informes técnicos, 724).

8. Glantz SA. Primer of biostatistics. $3^{\text {rd }}$ ed. New York: McGraw-Hill, 1992.

9. Hultman E. Nutritional effects on work performance. Am J Clin Nutr 1989;49:949-57.

10. Wilmore JH, Freund BJ. Nutritional enhancement of athletic performance. Nutr Abstr Rev 1984;54:1-6.

11. Wahlquist ML, Read RSD. Nutrition, metabolism and exercise. Aust J Sci Med Sport 1980;12:54-7.

12. Hu FB, Rimm E, Smith-Warner SA, Feskanich D, Stampfer MJ, Ascheiro A. Reproducibility and validity of dietary patterns assessed with a food-frequency questionnaire. Am J Clin Nutr 1999;69:243-9.
13. Caldarone G, Teanquilli C, Giampietro M. Assessment of the nutritional state of top level football players. In: Santilli G, editor. Sports Medicine applied to football, 1990:133-41.

14. Reilly T. Physiological aspects of soccer. Biol Sport 1994;11:3-20.

15. Clement DB, Sawchuk LL. Iron status and sports performance. Sports Med 1981;1:65-74.

16. Rodomski MW, Sabiston BH, Isoard P. Development sports anaemia in physically fit men after daily sustained submaximal exercise. Aviat Space Environ Med 1980; 51:41-5.

17. Paulev P, Jordal R, Pedersen N. Dermal excretion of iron in intensively training athletes. Clinica Chemica Acta 1983;127:19-27.

18. Siegal A, Hennekius C, Salomin H, Vanboeck B. Exercise related haematuria. JAMA 1979;241:391-2.

19. Halberg L, Magnusson B. The etiology of sports anaemia. Acta Med Scand 1984;216:145-8.

20. Pate RR. Sports anaemia: a review of the current literature. Physician Sports Med 1983;11:115-27. 